OPEN

SUBJECT AREAS:

OPTICS AND PHOTONICS

MATERIALS FOR OPTICS

Received

11 September 2013

Accepted

24 October 2013

Published

13 November 2013

Correspondence and requests for materials should be addressed to G.D. Igdaguanno@ nanogenesisgroup. com; giuseppe. daguanno@us.army. mil)

\section{Impedance matched thin metamaterials make metals absorbing}

\author{
N. Mattiucci' , M. J. Bloemer ${ }^{2}$, N. Aközbek' \& G. D'Aguanno'
}

\author{
${ }^{1}$ AEgis Tech., Nanogenesis Division 410 Jan Davis Dr, Huntsville, AL 35806, USA, ${ }^{2}$ Dept. of the Army, Charles M. Bowden Facility, \\ Redstone Arsenal, AL 35898, USA.
}

Metals are generally considered good reflectors over the entire electromagnetic spectrum up to their plasma frequency. Here we demonstrate an approach to tailor their absorbing characteristics based on the effective metamaterial properties of thin, periodic metallo-dielectric multilayers by exploiting a broadband, inherently non-resonant, surface impedance matching mechanism. Based on this mechanism, we design, fabricate and test omnidirectional, thin ( $<1$ micron), polarization independent, extremely efficient absorbers (in principle being capable to reach $\mathrm{A}>99 \%$ ) over a frequency range spanning from the UV to the IR. Our approach opens new venues to design cost effective materials for many applications such as thermo-photovoltaic energy conversion devices, light harvesting for solar cells, flat panel display, infrared detectors, stray light reduction, stealth and others.

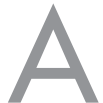

bsorbing materials are crucial in many applications such as thermo-photovoltaic energy conversion devices $^{1}$, light harvesting for solar cells ${ }^{2}$, flat panel displays ${ }^{3}$, stealth, and stray light reduction in imaging devices $^{4}$. In nature, black pigments are found in the compound eyes of most insects to reduce crosstalk . $^{5}$ Another case is the black color on the Papilio Ulysses butterfly wings that is due to a combination of black pigment and structured surface that traps the light ${ }^{6}$. Many venues have been proposed during recent years to achieve broadband, polarization-insensitive, omni-directional absorbers. Currently available broadband lowreflectance materials include gold black ${ }^{7}$, black nickels, including porous nickel phosphorus (NiP) ${ }^{8}$ and black paint. Black metals and paints tend to have rough, protruding surface features to trap the light causing it to undergo several reflections. A material with $15 \%$ reflectance can absorb $>99 \%$ of the energy after just three reflections. Black materials with rough surfaces such as anodized aluminum perform best at near normal incidence and degrade significantly for large angles ${ }^{4}$. Recently, successful demonstration of an extremely dark material has been made from low density nano-tube arrays ${ }^{9-11}$. However, fabrication of mechanically and environmentally durable nano-tube coatings over large areas is still a challenge and involves costly processes. Emerging new technologies have focused on absorption mechanisms based on the excitation of localized and/or surface plasmons on nano-patterned metal surfaces. For example, in Ref. 12 the authors experimentally demonstrate an ultrathin plasmonic absorber over the entire visible spectrum, in Ref. 13 the authors numerically study a broadband, 1-D metal grating with almost perfect absorption at $\lambda \sim 600 \mathrm{~nm}$, in Ref. 14 broadband absorption is predicted for all-metallic 1-D and 2-D gratings, in Ref. 15 an absorber based on light propagation in a metamaterial forming an effective "black-hole" is discussed, in Ref. 16 it is proposed a negative-index based, wide angle absorber for IR radiation, and in Ref. 17 broadband absorbers and selective emitters based on plasmonic Brewster metasurfaces are numerically investigated.

In this work we propose a viable, yet effective, alternative to achieve efficient absorbers spanning from the UV to the near IR by matching the impedance of a metamaterial to the impedance of air. The geometry of the multilayer stack under consideration is depicted in Fig. 1. It consists of an $N$-period, symmetric structure whose elementary cell is made of a metallic layer sandwiched between two identical dielectric layers. The metamaterial does not require surface features or nanopatterning and can be deposited over large areas with traditional deposition techniques.

In Ref. 18 we have shown that in general any stratification with a symmetric elementary cell, but not limited to a metallo-dielectric stratification, or a symmetric three-layer elementary cell described in Fig. 1, is equivalent to an effective bulk metamaterial, having the same physical thickness of the equivalent multilayer and univocally described by an effective characteristic impedance $\left(Z_{\text {eff }}\right)$ and the Bloch wave vector $\left(K_{B}\right)$ of the elementary cell. The expression of $Z_{e f f}, K_{B}$ and the free space characteristic impedance $\left(Z_{f_{s}}\right)$ are respectively: 
$\left[\text { Dielectric }\left(d_{D}\right) \backslash \text { Metal }\left(d_{M}\right) \backslash \text { Dielectric }\left(d_{D}\right)\right]^{N}$

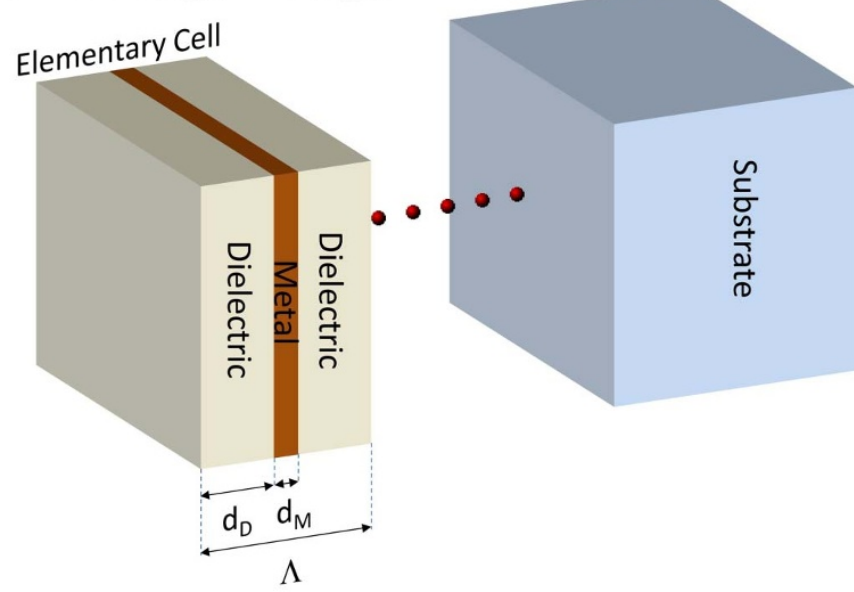

Figure 1 Generic metal/dielectric/metal symmetric elementary cell of period $\Lambda=2 d_{D}+d_{M}$. The overall structure consists of $\mathrm{N}$ repetition of the elementary cell grown on a substrate.

$$
\begin{aligned}
& \left\{\begin{array}{l}
\operatorname{Cos}\left(K_{B} \Lambda\right)=m_{11} \\
\left(\frac{Z_{\text {eff }}}{\omega}\right)^{2}=-\frac{m_{12}}{m_{21}} \\
\left(\omega Z_{\text {eff }}\right)^{2}=-\frac{m_{21}}{m_{12}}
\end{array}\right.
\end{aligned}
$$

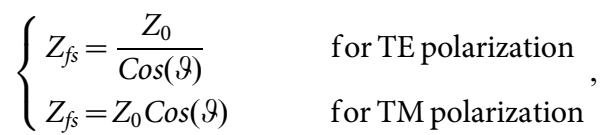

where $\Lambda$ is the period of the symmetric elementary cell, $\omega$ the frequency, $m_{i j}$ the elements of the transfer matrix of the elementary cell, $\mathrm{Z}_{0}$ the vacuum impedance, and $\vartheta$ the incident angle. Additional details of the derivation of Eqs.(1) and (2) are presented in the Methods. We note that our homogenization procedure, different from conventional approaches, does not rely on the approximation that the incident wavelength must be much longer than the dimension of the elementary cell. It maintains a general validity for any periodic stratification, not necessarily metallo-dielectric, characterized by a symmetric elementary cell ${ }^{18}$. The propagation length $\left(L_{P}\right)$, i.e. the distance for the intensity of an electromagnetic wave propagating inside the metamaterial to decay by a factor of $1 / e$, can be extracted from the imaginary part of the Bloch vector: $L_{P}=$ $\left(2 \operatorname{Im}\left(K_{B}\right)\right)^{-1}$. The propagation length $\left(L_{P}\right)$ together with the effective characteristic impedance $\left(Z_{\text {eff }}\right)$ are the key quantities to understand the absorption mechanism in these structures. Ideally, in order to achieve an efficient absorber we need a metamaterial that satisfies two requirements: first, it has to possess an effective characteristic impedance that matches the free space one for all incident angle and all polarization $\left(Z_{e f f}=Z_{f s}\right)$ and, second, it has to possess a very short propagation length, say few elementary cells. The first condition, $Z_{\text {eff }}$ $=Z_{f s}$, ensures that all the electromagnetic energy impinging the surface of the metamaterial enters into it with no reflections, while the second condition ensures that all the energy is efficiently absorbed within a few elementary cells. Bulk metals could in principle be good absorbers as they exhibit a consistent imaginary part of the permittivity, but actually they are not because, below the plasma frequency, the electromagnetic radiation practically cannot penetrate into them due to their high impedance mismatch, and thus they reflect most of the light. In what follows, using metamaterial concepts, we demonstrate both theoretically and experimentally a method to tailor their effective impedance and match it to the vacuum over a broad frequency range which can span the Vis and near IR.
For a metallo-dielectric stratification with the symmetric threelayer elementary cell described in Fig. 1, the effective impedance at normal incidence from Eq.(1) and (2) can be explicitly written as:

$\frac{Z_{\text {eff }}}{Z_{0}}=\frac{1}{n_{D}}$

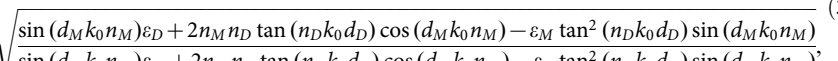

$\sqrt{\frac{\sin \left(d_{M} k_{0} n_{M}\right) \varepsilon_{M}+2 n_{M} n_{D} \tan \left(n_{D} k_{0} d_{D}\right) \cos \left(d_{M} k_{0} n_{M}\right)-\varepsilon_{D} \tan ^{2}\left(n_{D} k_{0} d_{D}\right) \sin \left(d_{M} k_{0} n_{M}\right)}{}}$,

where $n_{M}$ and $n_{D}$ are respectively the refractive index of the metal and dielectric layer, while $\varepsilon_{\mathrm{M}}$ and $\varepsilon_{\mathrm{D}}$ their respective relative electric permittivities. Here for simplicity we analyze theoretically the normal incidence case, nevertheless all the resulting considerations can be straightforwardly extended to any arbitrary incident conditions and polarizations. For thin metallic layers, i.e. $k_{0} d_{M}<<1$, a first order expansion of the trigonometric functions in (3) gives the following expression for the effective impedance:

$\frac{Z_{\text {eff }}}{Z_{0}}=\frac{1}{n_{D}} \sqrt{\frac{d_{M} k_{0} \varepsilon_{M} \tan ^{2}\left(n_{D} k_{0} d_{D}\right)-2 n_{D} \tan \left(n_{D} k_{0} d_{D}\right)-d_{M} k_{0} \varepsilon_{D}}{d_{M} k_{0} \varepsilon_{D} \tan ^{2}\left(n_{D} k_{0} d_{D}\right)-2 n_{D} \tan \left(n_{D} k_{0} d_{D}\right)-d_{M} k_{0} \varepsilon_{M}}}$.

Eq.(4) can be recast in a more convenient form as:

$$
\frac{Z_{\text {eff }}}{Z_{0}}=\frac{1}{n_{D}} \sqrt{\frac{f_{N}(x)}{f_{D}(x)}},
$$

where the numerator function $f_{N}(x)$ and the denominator function $f_{D}(x)$ are two parabolas respectively given by:

$$
\begin{aligned}
& f_{N}(x)=x^{2} \varepsilon_{M} \delta-2 n_{D} x-\varepsilon_{D} \delta, \\
& f_{D}(x)=x^{2} \varepsilon_{D} \delta-2 n_{D} x-\varepsilon_{M} \delta,
\end{aligned}
$$

with $x=\tan \left(n_{D} k_{0} d_{D}\right)$ and $\delta=k_{0} d_{M}<<1$. For the sake of simplicity, in the analysis that follows we neglect the imaginary part of the permittivity of the metal (as we will see later on, the results that stem from this analysis hold also when realistic metallic dispersions are used). Obviously, when the radicand of Eq.(5.a) is negative the effective impedance will be a pure imaginary number and therefore the metamaterial will show metallic-like behavior with $100 \%$ reflectivity and complete impedance mismatch, while in the case that the radicand of Eq.(5.a) is positive the effective impedance will be positive and the structure will show a dielectric-like behavior. In order to study the sign of the radicand, Eq.(5.a) can be rewritten, using Ruffini's theorem, as:

$$
\frac{Z_{e f f}}{Z_{0}}=\frac{1}{n_{D}} \sqrt{\frac{\varepsilon_{M}\left(x-x_{-N}\right)\left(x-x_{+N}\right)}{\varepsilon_{D}\left(x-x_{-D}\right)\left(x-x_{+D}\right)}},
$$

where the zeros of $f_{N}(x)$ and $f_{D}(x)$ are respectively given by:

$$
\begin{aligned}
& x_{ \pm N}=\frac{n_{D}}{\varepsilon_{M} \delta}\left(1 \pm \sqrt{1+\varepsilon_{M} \delta^{2}}\right), \\
& x_{ \pm D}=\frac{n_{D}}{\varepsilon_{D} \delta}\left(1 \pm \sqrt{1+\varepsilon_{M} \delta^{2}}\right) .
\end{aligned}
$$

For $\delta>1 / \sqrt{-\varepsilon_{M}}$ (Case I) life is rather dull, in fact the radicand of Eq.(6.a) will always be negative and the structure will show a metallic-like behavior. After all, this is the regime one would expect given the presence of metal layers. Luckily, things become much more interesting for $\delta<1 / \sqrt{-\varepsilon_{M}}$ (Case II). In this latter case, the radicand becomes positive when either $x_{-N}<x<x_{+N}$ or $x_{-D}<x<x_{+D}$ and in those intervals the structure will show a dielectric-like behavior In Fig. 2 we show a graphical representation that summarizes the results. 


$$
\frac{Z_{e f f}}{Z_{0}}=\frac{1}{n_{D}} \sqrt{\frac{f_{N}(x)}{f_{D}(x)}}
$$

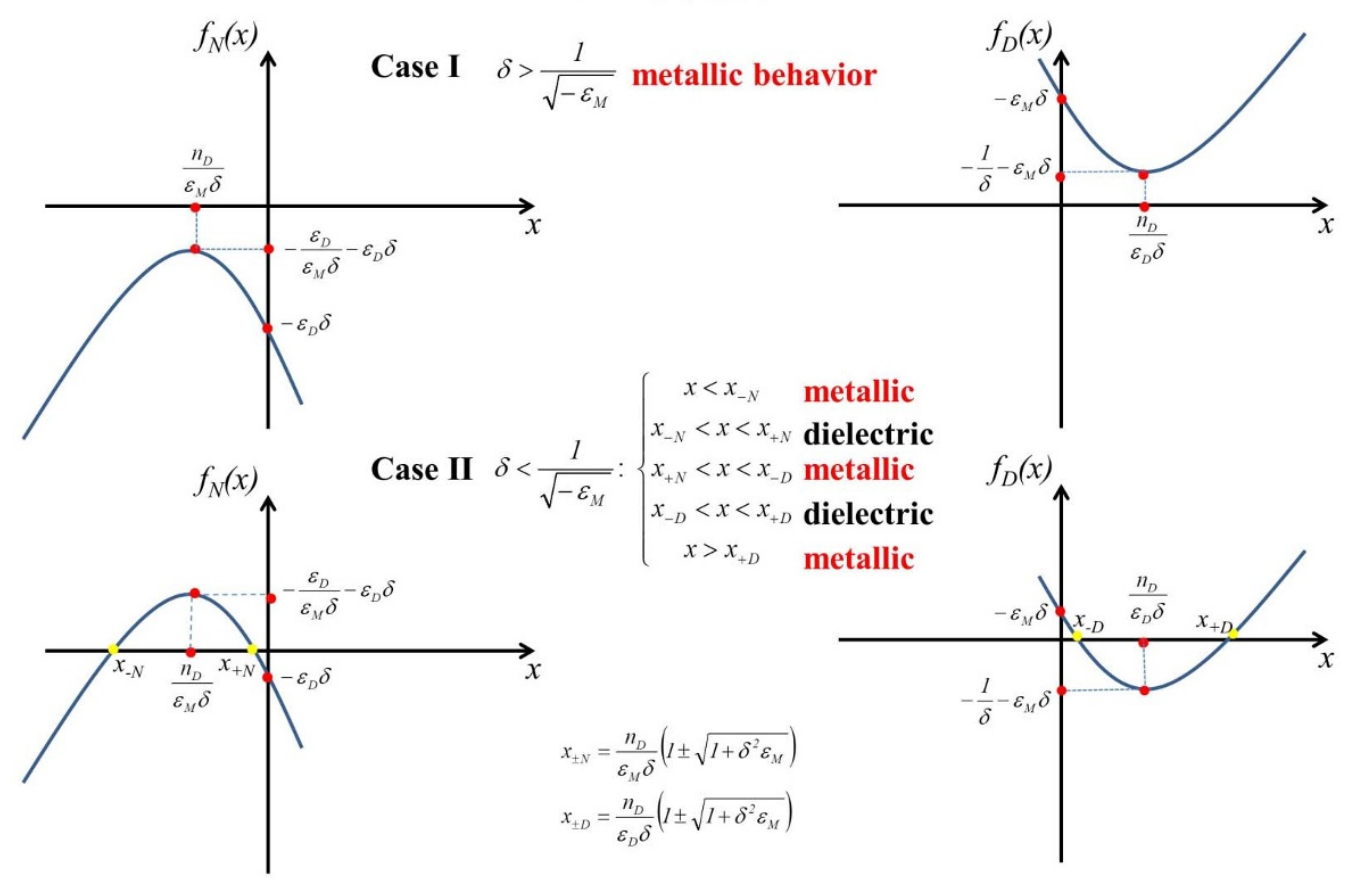

Figure $2 \mid$ Graphical representation of the study of the sign of the radicand of Eq.(5.a). In Case II bands of metallic-like behavior alternate with bands of dielectric-like behavior.

In particular, Case II of Fig. 2 shows the bands where the structure either behaves as a metal or as a dielectric. The two dielectric regions are bounded respectively by the zeroes and the poles of the effective impedance. Namely, the dielectric region $x_{-N}<x<x_{+N}$ is bounded by $Z_{\text {eff }} / Z_{0}=0$ (zeroes of $f_{N}(x)$ ), while the dielectric region $x_{-D}<x<x_{+D}$ is bounded by $Z_{e f f} / Z_{0}=\infty$ (zeros of $f_{D}(x)$ ). A further analysis of the effective dispersion of the conditions $Z_{\text {eff }} / Z_{0}=\infty$ and $Z_{\text {eff }} / Z_{0}=0$ shows that they can be approximately expressed as:

$$
\begin{gathered}
\frac{Z_{\text {eff }}}{Z_{0}}=\infty \begin{cases}d_{D}=-\frac{1}{2} \frac{\varepsilon_{M}}{\varepsilon_{D}} d_{M}+\frac{m}{2 n_{D}} \lambda & m=0,1,2, \ldots \\
d_{D}=-\frac{1}{2} d_{M}+\frac{2 m+1}{4 n_{D}} \lambda & m=0,1,2, \ldots\end{cases} \\
\frac{Z_{\text {eff }}}{Z_{0}}=0 \begin{cases}d_{D}=-\frac{1}{2} \frac{\varepsilon_{M}}{\varepsilon_{D}} d_{M}+\frac{2 m-1}{4 n_{D}} \lambda & m=1,2,3, \ldots \\
d_{D}=-\frac{1}{2} d_{M}+\frac{m}{2 n_{D}} \lambda & m=1,2,3, \ldots\end{cases}
\end{gathered}
$$

Let us now analyze in particular the dielectric region $x_{-D}<x<x_{+D}$, i.e. the one bounded by $Z_{\text {eff }} / Z_{0}=\infty$. The impedance at the center of this band is:

$$
\left(\frac{Z_{\text {eff }}}{Z_{0}}\right)_{x=\frac{n_{D}}{\varepsilon_{D} \delta}}=\sqrt{\frac{\frac{2}{\varepsilon_{D}}-\frac{\varepsilon_{M}}{\varepsilon_{D}^{2}}+\delta^{2}}{1+\varepsilon_{M} \delta^{2}}} .
$$

By solving Eq.(8) under the condition of perfect impedance matching with vacuum, we find:

$$
\left(\frac{Z_{e f f}}{Z_{0}}\right)_{x=\frac{n_{D}}{\varepsilon_{D}}}=1 \Leftrightarrow \varepsilon_{D}=\frac{1+\sqrt{1-\varepsilon_{M}\left(1+\varepsilon_{M} \delta^{2}-\delta^{2}\right)}}{1+\varepsilon_{M} \delta^{2}-\delta^{2}} .
$$

Eq.(9) dictates what is the best choice of the permittivity of the dielectric material for perfect impedance matching in the dielectric band. Therefore, not only our structure possesses bands of dielectric-like behavior, but, in principle, it is also possible to match its impedance to the vacuum by a proper choice of the dielectric material, no matter how many metal layers the structure is made of. The structure could contain hundreds of metal layers and still be impedance matched with vacuum!

In Fig. 3 we plot, as an example, $\varepsilon_{D}$ vs. $\lambda / \lambda_{p}$ according to (9) for metal layers with different thickness whose dispersion we model by using a generic, free-electron gas, lossless Drude model $\varepsilon_{M}=1-\left(\lambda / \lambda_{p}\right)^{2}\left(\lambda_{p}=2 \pi \mathrm{c} / \omega_{\mathrm{p}}\right.$ is the metal plasma wavelength).

In Fig. 3, it can be seen that the values of the permittivity of the dielectric materials necessary to achieve perfect impedance matching are within the range of the permittivity of many dielectric and/or semiconductor materials. For example, in Fig. 4 we show the reflectance at normal incidence $R=\left|1-\left(Z_{\text {eff }} / Z_{0}\right) / 1+\left(Z_{\text {eff }} / Z_{0}\right)\right|^{2}$ numerically calculated in the plane $\left(\mathrm{d}_{\mathrm{D}} / \lambda_{\mathrm{p}}, \lambda / \lambda_{\mathrm{p}}\right)$ for $\mathrm{d}_{\mathrm{M}} / \lambda_{\mathrm{p}}=1 / 30$ and $\varepsilon_{\mathrm{D}}=2.25$. As expected from the previous study, the structure

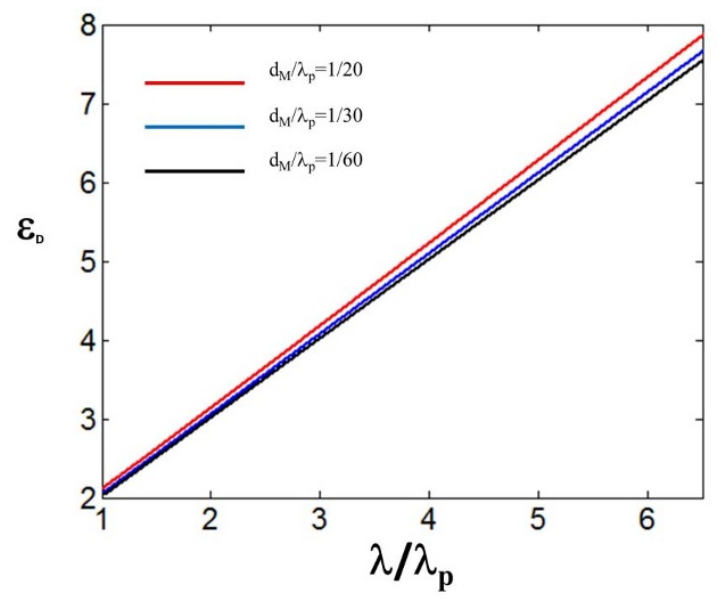

Figure $3 \mid \varepsilon_{D}$ vs. $\lambda / \lambda_{p}$ according to (9) for a lossless free electron gas. 


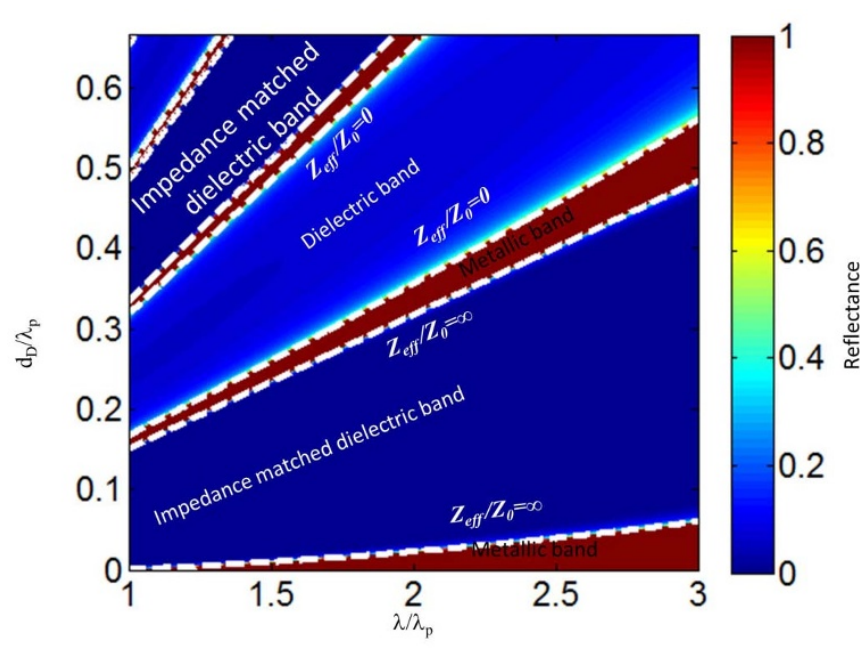

Figure $4 \mid$ Reflectance in the $\left(d_{D} / \lambda_{p}, \lambda / \lambda_{p}\right)$ for $d_{M} / \lambda_{p}=1 / 30$ and $\varepsilon_{D}=$ 2.25. The metal layer dispersion is described with a generic, free-electron gas, lossless Drude model.

shows two bands of dielectric-like behavior. The one that is bounded by the poles of the effective impedance shows perfect impedance matching. The white dashed lines represent the dispersion of the poles and the zeroes of the effective impedance calculated according to (7).

Now that we have analyzed in detail the properties of the effective impedance of our metamaterial and the impedance matching condition, we come to the analysis of the propagation length $\left(\mathrm{L}_{\mathrm{P}}\right)$. As already mentioned in the introduction, a good absorber should be characterized by a short propagation length. At normal incidence for a semi-infinite (s-i) structure the absorption is given by the well-knownformula: $A_{s-i}=4 \operatorname{Re}\left(Z_{\text {eff }} / Z_{0}\right) /\left(\left|Z_{\text {eff }} / Z_{0}\right|^{2}+2 \operatorname{Re}\left(Z_{\text {eff }} / Z_{0}\right)+1\right)$. By expanding the Bloch vector at the first order for thin metal layers, it can be demonstrated by simple mathematical manipulations that, at the center of the pass band of the Bloch vector, i.e. for $\cos \left(\mathrm{K}_{\mathrm{B}} \Lambda\right)=$ $0, L_{P}$ can be approximated as:

$$
\frac{L_{P}}{\Lambda}=\frac{n_{D}}{\operatorname{Im}\left(\varepsilon_{M}\right) k_{0} d_{M}},
$$

Eq.(10) tells us that the propagation length is inversely proportional to the imaginary part of the metal permittivity (dissipation), as one may expect, and directly proportional to the refractive index of the dielectric material. In order to have an efficient absorber a metal with high dissipation properties, such as $\mathrm{Cu}$ for example, would be desirable in conjunction with a low refractive index dielectric material, ideally as close as possible to vacuum. So far we have concentrated for the sake of generality on metals with nonspecific dispersion properties. We want now provide two examples in which metals with realistic dispersion properties are used. In particular, we concentrate on copper $(\mathrm{Cu})$ and silver $(\mathrm{Ag})$. In Fig. 5, we plot the bulk dispersion relations of the two metals, as reported in the book of Palik $^{19}$, it is worthwhile to note that copper shows intrinsic better absorption properties, i.e. higher imaginary part of the permittivity, with respect to Ag. In order to be as realistic as possible, we have also taken into account the increased resistivity of the thin metallic layers when their thickness becomes comparable or shorter than the electron mean free path through the Fuchs-Sondhemeir theory ${ }^{20,21}$. More details about the procedure used to evaluate the dispersion of the thin metallic layers can be found in the Methods.

In Fig. 6 and 7 we show the numerically calculated absorption at normal incidence for a semi-infinite structure $\left(A_{s-i}\right)$ and $\mathrm{L}_{\mathrm{p}} / \Lambda$ in the $\left(\lambda, d_{D}\right)$ plane, respectively for a $n_{D}\left(d_{D}\right) / C u(10 n m) / n_{D}\left(d_{D}\right)$

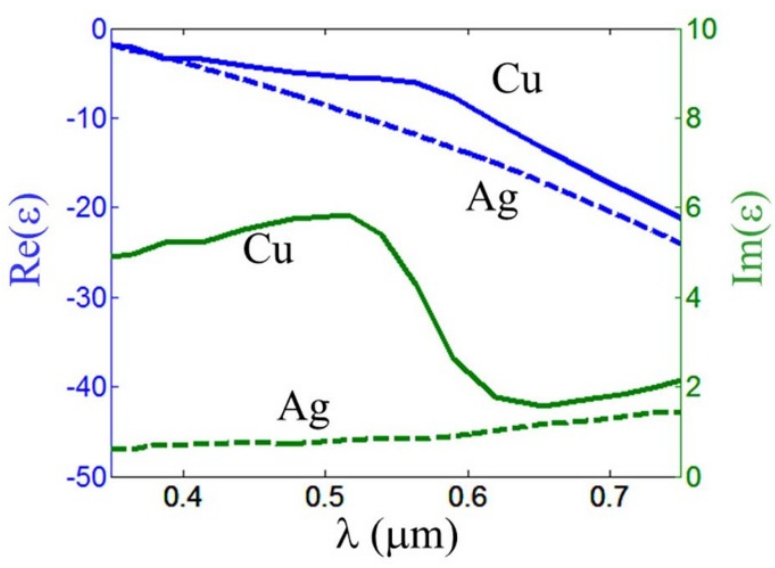

Figure 5 Bulk dispersions for $\mathrm{Ag}$ and $\mathrm{Cu}$ as reported in the book of Palik ${ }^{19}$.

elementary cell and for a $\mathrm{n}_{\mathrm{D}}\left(\mathrm{d}_{\mathrm{D}}\right) / \operatorname{Ag}(10 \mathrm{~nm}) / \mathrm{n}_{\mathrm{D}}\left(\mathrm{d}_{\mathrm{D}}\right)$ with $\mathrm{n}_{\mathrm{D}}=$ $1.52\left(\mathrm{SiO}_{2}\right)$. In both Fig. 6(a) and Fig. 7(a) broad (non-resonant) regions of impedance matching and high absorption $(\mathrm{A}>99 \%)$ are evident. Those regions are approximately bounded by the poles of the effective impedance (white dashed lines), consistent with the results of the previous study summarized in Fig. 2. Bands of dielectric-like behavior are also present and they are approximately bounded by the zeroes of the effective impedance, as expected. Note that, although in this case we fully take into account the metal dissipation without neglecting the imaginary part of its permittivity, nevertheless the general picture previously described still holds, as expected.

Fig. $6(\mathrm{~b})$ and $7(\mathrm{~b})$ show $\mathrm{L}_{\mathrm{P}} / \Lambda$ in the $\left(\lambda, \mathrm{d}_{\mathrm{D}}\right)$ plane. The yellow line represents here the center of the pass band calculated according to the condition $\cos \left(\mathrm{K}_{\mathrm{B}} \Lambda\right)=0$. It is seen that $L_{P}$ in the $\mathrm{Ag}$ case is much longer than the $\mathrm{Cu}$ case.

In view of Eq.10, the reason for the different performances is ascribed to the intrinsic better absorption properties of $\mathrm{Cu}$, i.e. higher imaginary part of the permittivity, with respect to $\mathrm{Ag}$, as it is seen from the bulk dispersion properties of both materials reported in Fig. 5. Fig. 6(c) and 7(c) compare $\mathrm{L}_{\mathrm{P}} / \Lambda$ calculated at the center of the pass band according to the exact formula $L_{P}=\left(2 \operatorname{Im}\left(K_{B}\right)\right)^{-1}$ and according to (10).

The results on the propagation length suggest that the choice of the particular kind of metal is essential to obtain efficient absorption in short distances. While in fact, in the examples made, both structures ensure a high degree of impedance matching, the Cu-based structure is much more efficient in absorbing the e.m. radiation than the Ag-based structure. This means that, based on the respective propagation lengths, a 6-period $\mathrm{Cu}$-based structure would be highly absorbing whereas the same structure made of Ag would be highly transparent. Although here we are interested in increasing the absorption properties of the structure, it is nevertheless interesting to note the great versatility of the impedance matching mechanism that we have elucidated: it makes possible to pass from a highly absorbing structure to a highly transparent structure just by a proper choice of the particular kind of metal.

\section{Results}

Based on the theoretical study presented in the previous section, we have fabricated two samples: sample1 and sample2 (see Methods for the fabrication details). In both cases the elementary cell is a three layer stratification with $10 \mathrm{~nm}$ of $\mathrm{Cu}$ sandwiched between two layers of $\mathrm{SiO}_{2} 30 \mathrm{~nm}$ thick. Sample1 is made of 6 repetitions of the elementary cell for a total of $\mathrm{L} \sim 3 L_{p}$, sample 2 is made of 10 repetitions of the elementary cell for a total length $\mathrm{L} \sim 5 L_{p}$. Both samples have been grown on a quartz substrate. The transmittance $(\mathrm{T})$ of the two 

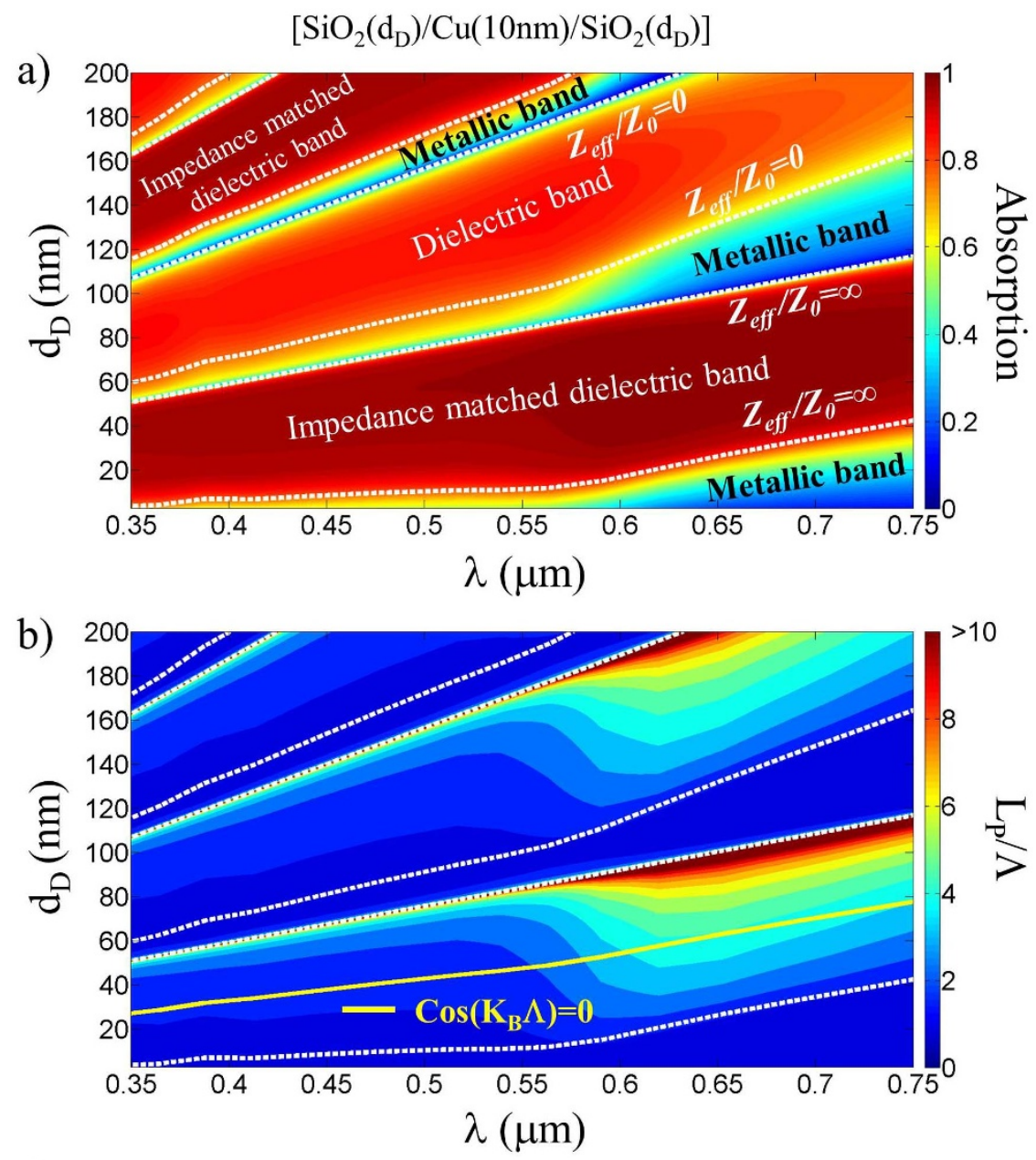

c)

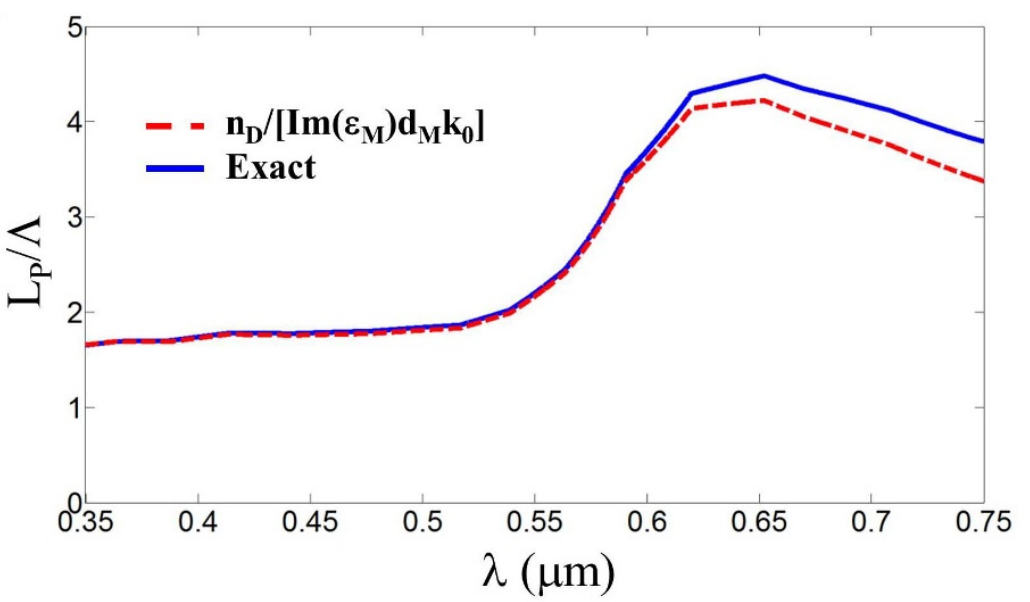

Figure 6 (a) Numerically calculated absorption of the semi-infinite structure in the $\left(\lambda, d_{D}\right)$ plane for a $n_{D}\left(d_{D}\right) / C u(10 \mathrm{~nm}) / n_{D}\left(d_{D}\right)$ elementary cell with $\mathrm{n}_{\mathrm{D}}=1.52\left(\mathrm{SiO}_{2}\right)$. The white dashed lines represent the poles and the zeroes of the effective impedance calculated according to $(7)$. Bands of impedance matching and almost unitary absorption bounded by the poles of the effective impedance are visible, as predicted. $(b) L_{P} / \Lambda$ in the $\left(\lambda, d_{D}\right)$ plane. The yellow line represents here the center of the pass band calculated according to the condition $\cos \left(\mathrm{K}_{\mathrm{B}} \Lambda\right)=0$. (c) $\mathrm{L}_{\mathrm{P}} / \Lambda$ vs. $\lambda$ calculated at the center of the pass band according to the exact formula $L_{P}=\left(2 \operatorname{Im}\left(K_{B}\right)\right)^{-1}$ and according to (10).

samples has been measured between $0^{\circ}$ and $70^{\circ}$ over a wide spectral range $(300 \mathrm{~nm}$ up to $800 \mathrm{~nm}$ ) while the reflectance $(\mathrm{R})$ has been measured between $20^{\circ}$ and $70^{\circ}$ over the same spectral range (see Methods for measurements details). In Fig. 8 and Fig. 9 we report (left panel) the measured average transmittance $(\mathrm{T})$ and reflectance (R) (the average has been taken over TE and TM polarization) as well as the derived absorption $(\mathrm{A}=1-\mathrm{R}-\mathrm{T})$ for sample1 and sample2 respectively in the $(\lambda, \vartheta)$ plane, in comparison with the same quantities as predicted by the theory (right panel). A close look at Fig. 8 and Fig. 9 reveals that the reflectance from the two samples is almost identical, while the transmittance is slightly higher for sample 1 and scales approximately as $T \sim \exp \left(-L / L_{p}\right)$ in agreement with the theoretical predictions based on the propagation length analysis. As expected, both samples show high degree of polarization independent absorption $(>90 \%)$, over a wide range of incident angles and a spectral band extending from UV to near IR. Note also that the first 

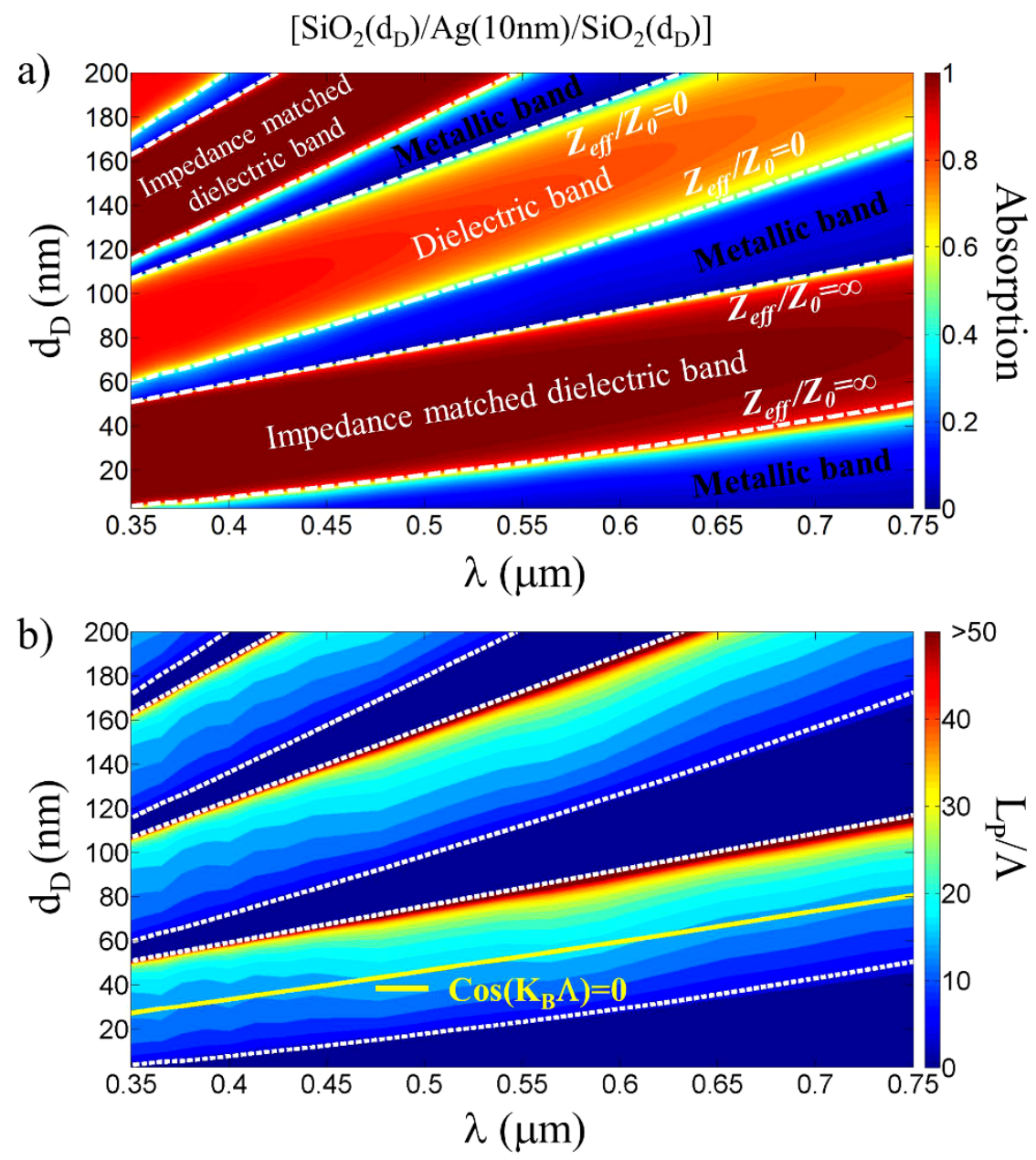

c)

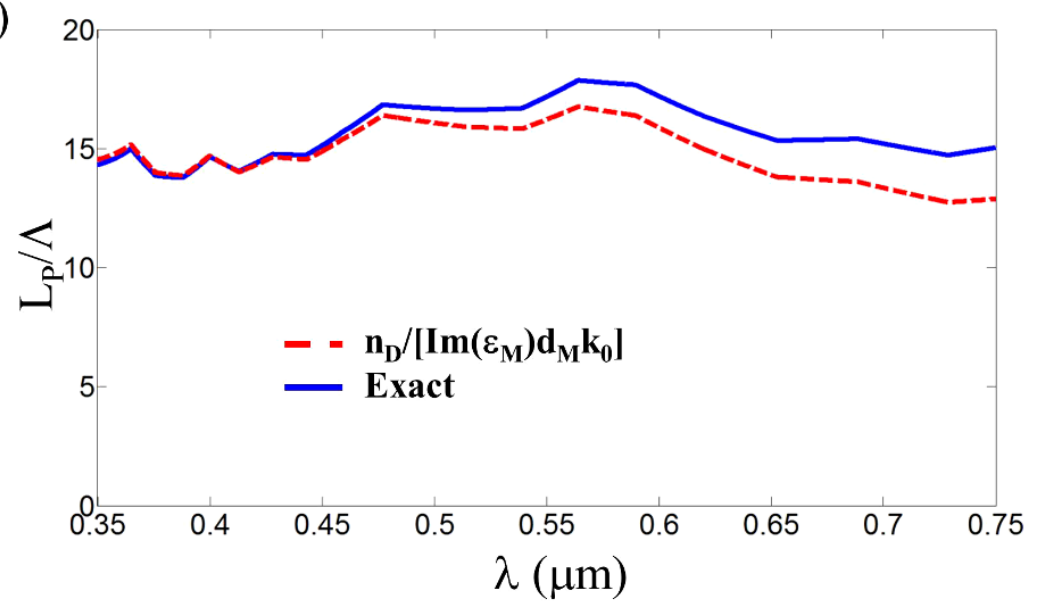

Figure $7 \mid$ Same as in Fig. 6 for the $n_{D}\left(d_{D}\right) / A g(10 n m) / n_{D}\left(d_{D}\right)$ elementary cell.

sample has a total length of only $420 \mathrm{~nm}$ and a total metal thickness of just $60 \mathrm{~nm}$. The experimental absorption near $750 \mathrm{~nm}$ is larger than predicted by theory and we attribute this to the possibility that the $10 \mathrm{~nm}$ thick $\mathrm{Cu}$ film is below the percolation threshold which could induce losses due to the excitation of localized surface plasmons.

\section{Discussion}

We have reported a practical and simple way to achieve broadband, omnidirectional, polarization-insensitive absorption using periodic, thin, metallo-dielectric, multilayers in their metamaterial operation regime. Those structures can be easily fabricated over large areas at low cost using standard sputtering or thermal evaporation techniques $^{22}$. We remark that, although in this work we have focused on $\mathrm{SiO}_{2} / \mathrm{Cu}$ multilayers for absorbing purposes, different combination of metal/dielectric materials can be exploited. As an example, in Fig. 10 we present the theoretical results for an $\mathrm{N}=10$-period structure whose elementary cell is made of a tungsten (W) layer $5 \mathrm{~nm}$ thick sandwiched between two $\mathrm{Ta}_{2} \mathrm{O}_{5}$ layers $75 \mathrm{~nm}$ thick. In this case the structure shows an ultra-wide band of abosrption ranging from $300 \mathrm{~nm}$ to $2.5 \mu \mathrm{m}$.

The possible applications for these structures are innumerable. Absorbing materials structured at the nanoscale level are a fundamental part in the design of any efficient thermo-photovoltaic 


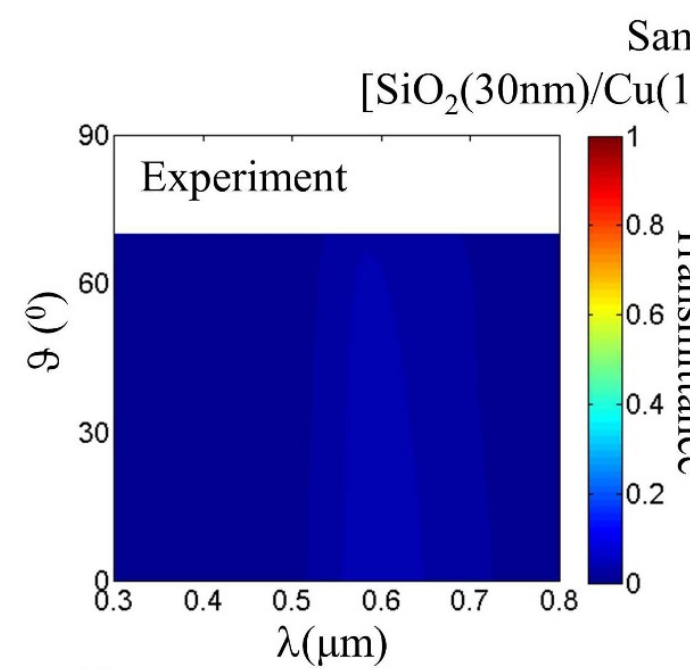

Sample 1
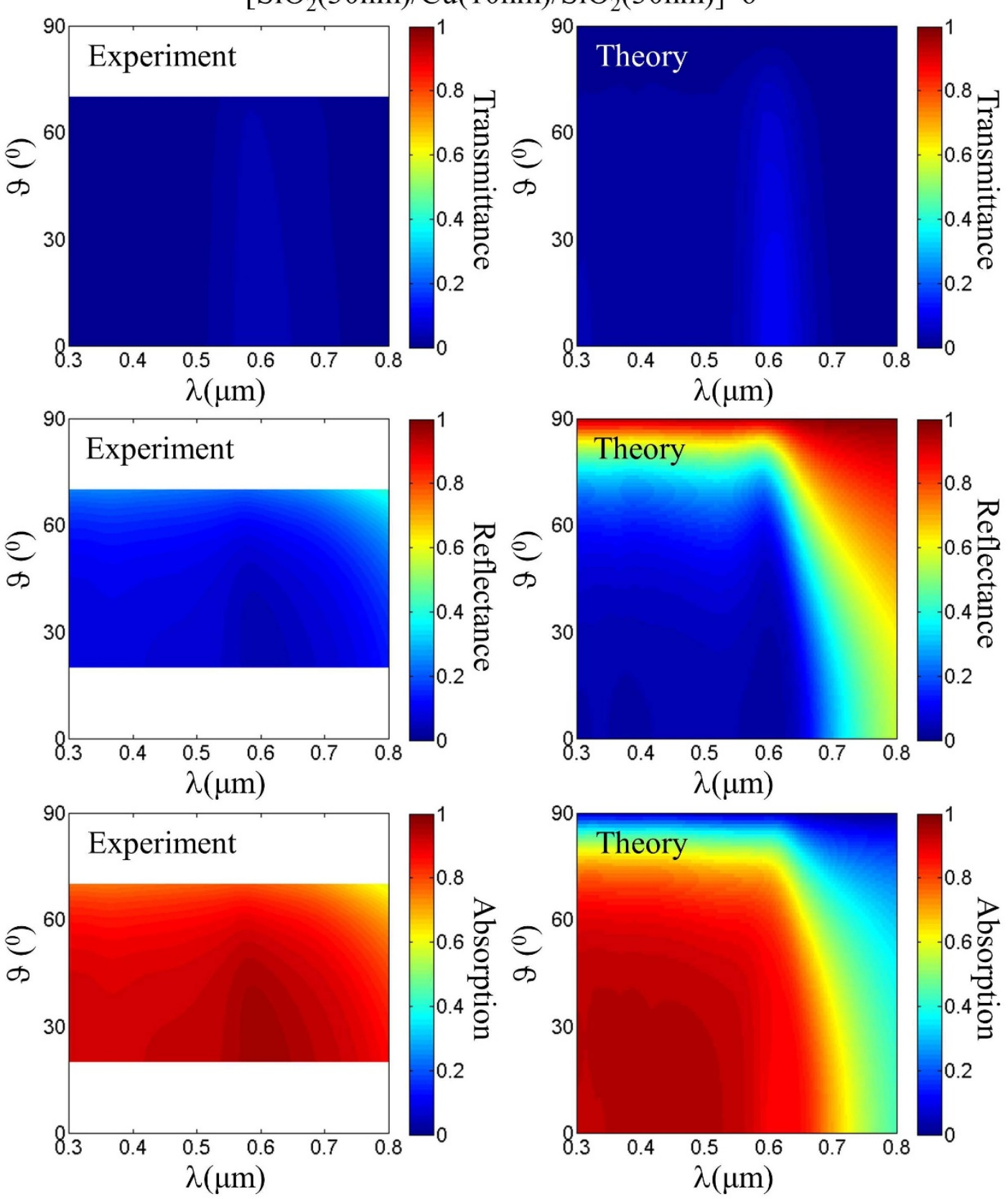

Figure $8 \mid$ (Left Panel) Average transmittance, reflectance and absorption measured for sample 1 in the $(\lambda, \vartheta)$ plane. (Right panel) Average transmittance, reflectance and absorption numerically calculated.

energy conversion device or solar cell. We also remark that the mechanism exploited in this work is based on a non-resonant impedance matching phenomenon which is therefore independent of the particular nature of the substrate. This makes possible, for example, to use our thin metamaterial to cover bulky metallic structures to eliminate electromagnetic reflection over an extremely broad frequency range from UV to almost mid-IR. As an example, we have grown sample 2 on a reflecting surface made of an optically thick silver layer. A picture of the sample and measured absorption is shown in Fig. 11.

Moreover, the non-resonant nature of the phenomenon also allows us to use these metamaterials in conjunction with other known absorbing configurations, such the classical Salisbury-mirror-like configuration, i.e. antireflection coating/absorber/mirror, to further enhance their performance.

We also mention that the same approach that here we have employed to obtain efficient absorbers metamaterials for the visible and near-IR could be employed as well to obtain efficient absorbers in the mid-IR by using polar material such as $\mathrm{SiC}$, for example.

\section{Methods}

Metamaterial description of multilayers. The fields at the input and output surface of a single layer material (a Fabry-Perot etalon) are linked to each other through a matrix relation that contains information on the angle of incidence by the

longitudinal momentum $\mathrm{k}_{\mathrm{z}}$ of the impinging wave, the material properties $\varepsilon$ and $\mu$, and the layer thickness $\mathrm{d}^{23}$ (see Fig. 12): 


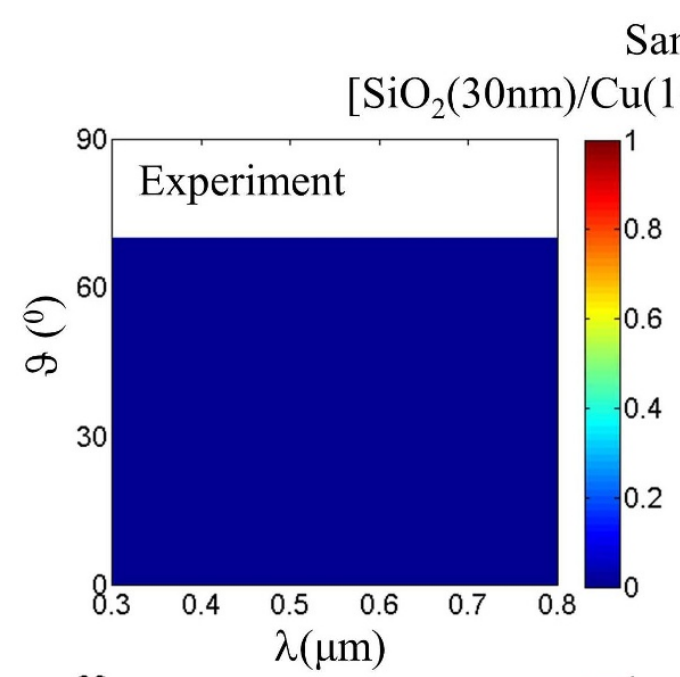

Sample 2
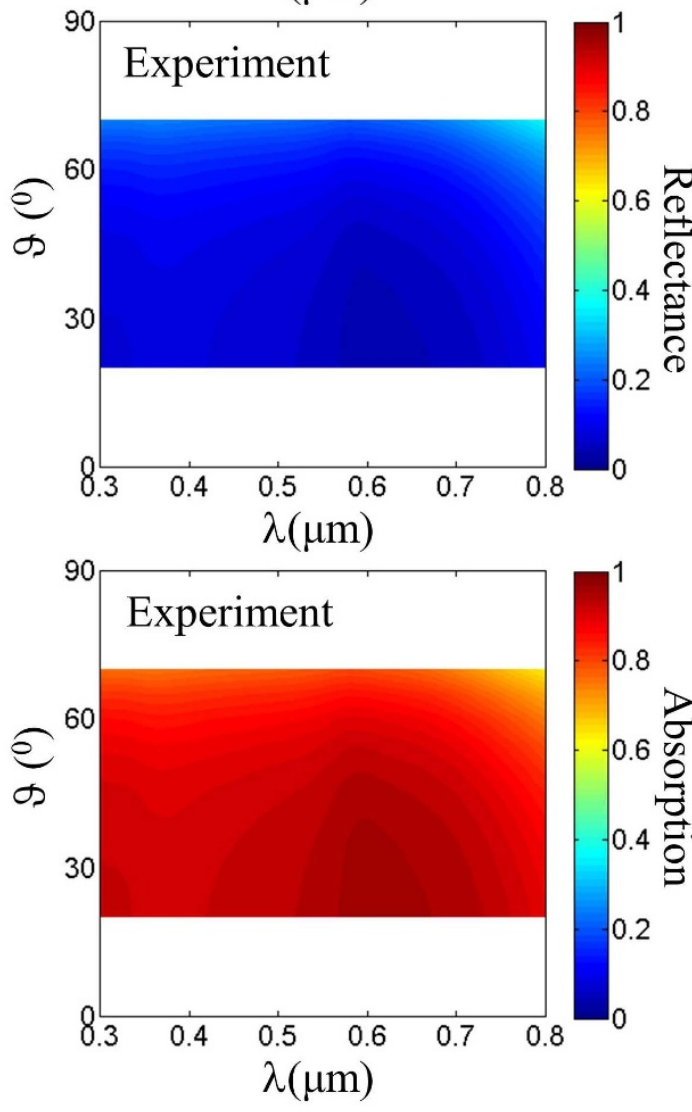

Figure 9 Same as in Fig. 8 for sample 2.

$$
\left(\begin{array}{c}
\Psi_{\text {Out }} \\
\varphi_{\text {Out }}
\end{array}\right)=\left(\begin{array}{cc}
\operatorname{Cos}\left(\mathrm{k}_{\mathrm{z}} \mathrm{d}\right) & \frac{\alpha}{\mathrm{k}_{\mathrm{z}}} \operatorname{Sin}\left(\mathrm{k}_{\mathrm{z}} \mathrm{d}\right) \\
-\frac{\mathrm{k}_{\mathrm{z}}}{\alpha} \operatorname{Sin}\left(\mathrm{k}_{\mathrm{z}} \mathrm{d}\right) & \operatorname{Cos}\left(\mathrm{k}_{\mathrm{z}} \mathrm{d}\right)
\end{array}\right)\left(\begin{array}{c}
\Psi_{\text {In }} \\
\varphi_{\text {In }}
\end{array}\right) .
$$

For TE polarization, $\alpha=\mu, \Psi=\mathrm{E}, \varphi=(1 / \mu) \mathrm{dE} / \mathrm{dz}$. For TM polarization, $\alpha=\varepsilon, \Psi=$ $\mathrm{H}$ and $\varphi=(1 / \varepsilon) \mathrm{dH} / \mathrm{dz}$. In a cascade of layers, the fields at the input and output interface are still related each other through a matrix relation. In this case the transfer matrix of the whole structure is the product of all the single layer transfer matrix. If we consider an elementary cell of length $\Lambda=\sum \mathrm{d}_{\mathrm{i}}$ we have:

$$
\begin{aligned}
\left(\begin{array}{c}
\Psi_{\text {Out }} \\
\varphi_{\text {Out }}
\end{array}\right) & =\prod_{\text {Elemetary Cell }}\left(\begin{array}{cc}
\operatorname{Cos}\left(\mathrm{k}_{\mathrm{z}}^{\mathrm{i}} \mathrm{d}_{\mathrm{i}}\right) & \frac{\alpha_{i}}{\mathrm{k}_{\mathrm{z}}^{\mathrm{S}}} \operatorname{Sin}\left(\mathrm{k}_{\mathrm{z}}^{\mathrm{i}} \mathrm{d}_{\mathrm{i}}\right) \\
-\frac{\mathrm{k}_{\mathrm{z}}^{\mathrm{i}}}{\alpha_{i}} \operatorname{Sin}\left(\mathrm{k}_{\mathrm{z}}^{\mathrm{i}} \mathrm{d}_{\mathrm{i}}\right) & \operatorname{Cos}\left(\mathrm{k}_{\mathrm{z}}^{\mathrm{i}} \mathrm{d}_{\mathrm{i}}\right)
\end{array}\right)\left(\begin{array}{c}
\Psi_{I n} \\
\varphi_{I n}
\end{array}\right) \\
& =\left(\begin{array}{cc}
m_{11}^{\Lambda} & m_{12}^{\Lambda} \\
m_{21}^{\Lambda} & m_{221}^{\Lambda}
\end{array}\right)\left(\begin{array}{c}
\Psi_{I n} \\
\varphi_{I n}
\end{array}\right)
\end{aligned}
$$
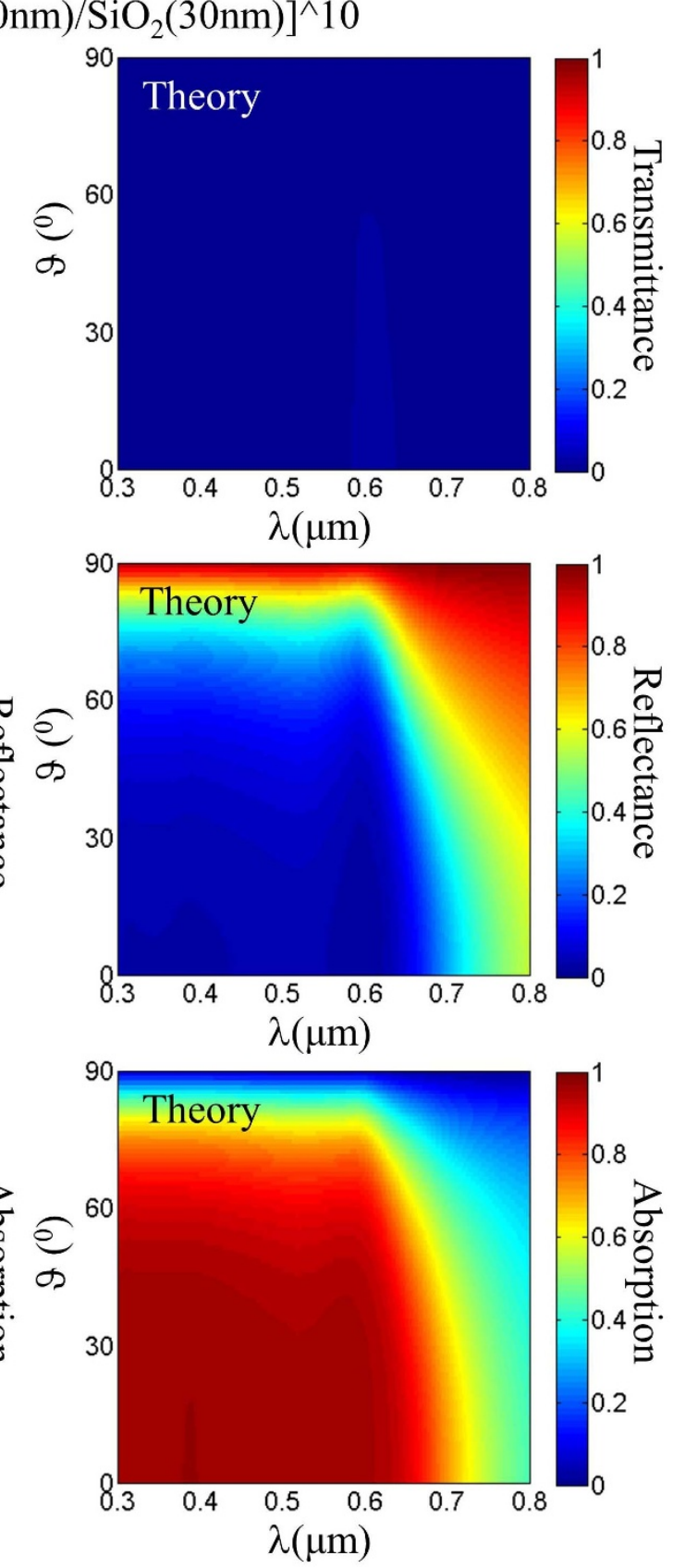

where $d_{i}$ is the thickness of the $i^{\text {th }}$ layer, $k_{z}^{i}$ is the longitudinal component of the $k$ vector in the $\mathrm{i}^{\text {th }}$ layer, $\alpha_{\mathrm{i}}=\mu_{\mathrm{i}}$ for TE polarization or $\alpha_{\mathrm{i}}=\varepsilon_{\mathrm{i}}$ for TM polarization, being $\mu_{\mathrm{i}}$ and $\varepsilon_{\mathrm{i}}$ the bulk magnetic permeability and the electric permittivity of the $\mathrm{i}^{\text {th }}$ layer. For a symmetric structure ${ }^{18}$ it is possible to define $\mathrm{k}_{\mathrm{z}}^{\text {eff }}$ and $\alpha_{\mathrm{eff}}$ so that:

$$
\left(\begin{array}{cc}
\operatorname{Cos}\left(\mathrm{k}_{\mathrm{z}}^{\text {eff }} \Lambda\right) & \frac{\alpha_{e f f}}{\mathrm{k}_{\mathrm{z}}^{\text {eff }}} \operatorname{Sin}\left(\mathrm{k}_{\mathrm{z}}^{\text {eff }} \Lambda\right) \\
-\frac{\mathrm{k}_{\mathrm{z}}^{\text {eff }}}{\alpha_{\text {eff }}} \operatorname{Sin}\left(\mathrm{k}_{\mathrm{z}}^{\text {eff }} \Lambda\right) & \operatorname{Cos}\left(\mathrm{k}_{\mathrm{z}}^{\text {eff }} \Lambda\right)
\end{array}\right)=\left(\begin{array}{cc}
m_{11}^{\Lambda} & m_{12}^{\Lambda} \\
m_{21}^{\Lambda} & m_{221}^{\Lambda}
\end{array}\right)
$$

Note that in general Eq. (13) provides 4 equations for 2 unknown quantities and in general there is no solution, unless the elementary cell is symmetric.

In Eq. (13), $\alpha_{\text {eff }}=\mu_{\text {eff }}$ (effective permeability) for TE polarization, or $\alpha_{\text {eff }}=\varepsilon_{\text {eff }}$ (effective permittivity) for TM polarization. $\mathrm{k}_{\mathrm{z}}^{\text {eff }}$ is the longitudinal component of the $\mathrm{k}$ vector in the effective medium: $\mathrm{k}_{\mathrm{z}}^{\text {eff }}=K_{B}=\mathrm{n}_{\text {eff }} \sqrt{(\omega / \mathrm{c})^{2}-\left(\mathrm{k}_{\mathrm{x}} / \mathrm{n}_{\text {eff }}\right)^{2}}$ where, $\mathrm{k}_{\mathrm{x}}$ is the transverse component of the $\mathrm{k}$ vector, that is continuous through the interfaces. $\mathrm{k}_{\mathrm{z}}^{\text {eff }}$ is also equal to the Bloch vector of the structure ${ }^{18}$. 

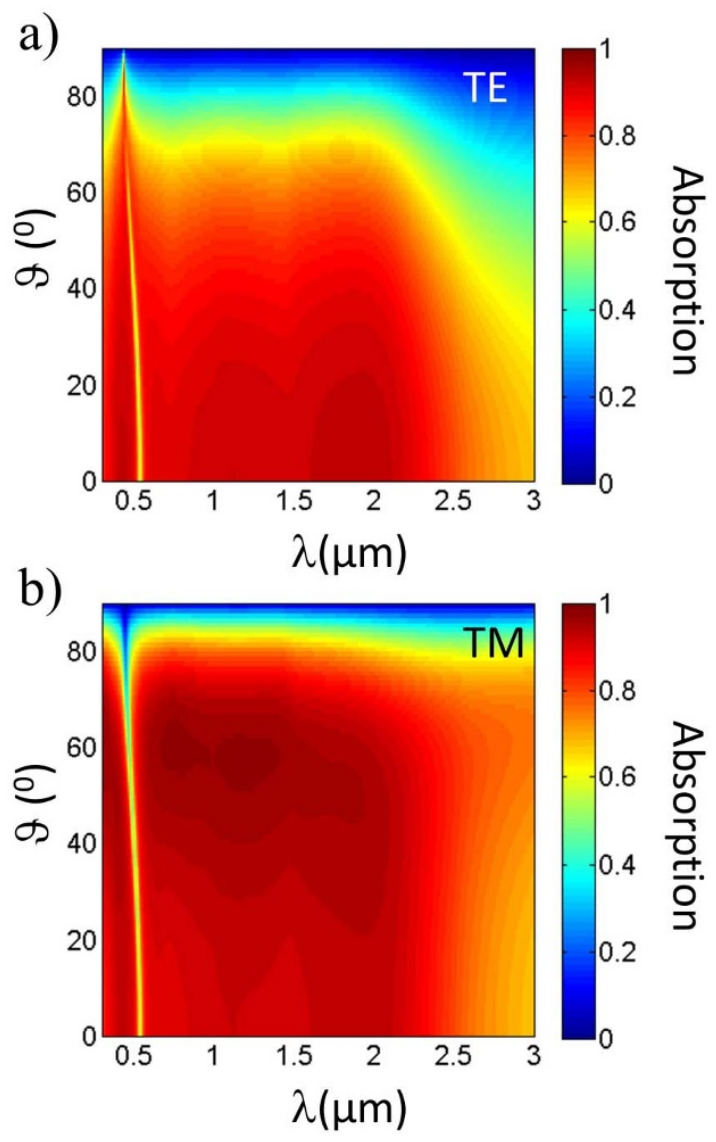

Figure $10 \mid$ Absorption calculated for a 10 period $\mathrm{Ta}_{2} \mathrm{O}_{5}(75 \mathrm{~nm}) /$ $\mathrm{W}(5 \mathrm{~nm}) / \mathrm{Ta}_{2} \mathrm{O}_{5}(75 \mathrm{~nm})$ structure.

From Eq. (13) we can derive

$$
\left\{\begin{array}{l}
\left(\frac{\alpha_{e f f}}{\mathrm{k}_{\mathrm{z}}^{\mathrm{eff}}}\right)^{2}=-\frac{m_{12}^{\Lambda}}{m_{21}^{\Lambda}} \\
\operatorname{Cos}\left(\mathrm{k}_{\mathrm{z}}^{\text {eff }} \Lambda\right)=m_{11}^{\Lambda}=m_{22}^{\Lambda}
\end{array}\right.
$$

The characteristic impedance in a generic medium takes the following expressions ${ }^{24}$ :

$$
\left\{\begin{array}{ll}
Z=\sqrt{\frac{\mu}{\varepsilon}} \frac{k}{k_{z}} & \text { for TE polarization } \\
Z=\sqrt{\frac{\mu}{\varepsilon} \frac{k_{z}}{k}} & \text { for TM polarization }
\end{array} .\right.
$$

Eq.(1) follows from Eq.(14) and (15). Finally, for N repetition of the elementary cell the transfer matrix is given by:

$$
\left(\begin{array}{ll}
m_{11}^{N \Lambda} & m_{12}^{N \Lambda} \\
m_{21}^{N \Lambda} & m_{22}^{N \Lambda}
\end{array}\right)=\left(\begin{array}{cc}
\operatorname{Cos}\left(\mathrm{k}_{\mathrm{z}}^{\mathrm{eff}} N \Lambda\right) & \frac{\alpha_{e f f}}{\mathrm{k}_{\mathrm{z}}^{\mathrm{eff}}} \operatorname{Sin}\left(\mathrm{k}_{\mathrm{z}}^{\mathrm{eff}} N \Lambda\right) \\
-\frac{\mathrm{k}_{\mathrm{z}}^{\mathrm{eff}}}{\alpha_{e f f}} \operatorname{Sin}\left(\mathrm{k}_{\mathrm{z}}^{\mathrm{eff}} N \Lambda\right) & \operatorname{Cos}\left(\mathrm{k}_{\mathrm{z}}^{\mathrm{eff}} N \Lambda\right)
\end{array}\right)
$$

In other words, the $\mathrm{N}$-period structure is equivalent to a bulk of the same effective medium of the elementary cell and having the same length $\mathrm{L}=\mathrm{N} \Lambda$ of the $\mathrm{N}$-period structure itself: i.e. the dispersion properties of the single, symmetric elementary cell and the dispersion of the N-period structure made of the same elementary cell are identical.

Dispersion properties of thin metallic layers. Metal layers with dimensions on the order of the electron mean-free path $(l)$ or less, exhibit higher resistivity than bulk conductors. The effect was first observed experimentally by Thomson in $1901^{25}$ and later theoretically explained by Fuchs in $1938^{20}$ and Sondheimer in $1952^{21}$. According to the Fuchs-Sondheimer theory, the thin layer conductivity $\left(\sigma_{\text {thin }}\right)$ is related to the bulk conductivity $(\sigma)$, through a function of the layer thickness $(\mathrm{d})$ over the electron mean-free path $(\xi=\mathrm{d} / l)$ :

$$
\frac{\sigma}{\sigma_{\text {thin }}}=\frac{\Phi(\xi)}{\xi}
$$

with

$$
\frac{1}{\Phi(\xi)}=\frac{1}{\xi}-\frac{3}{4}\left(1-\frac{1}{12} \xi^{2}\right) E i(-\xi)-\frac{3}{8 \xi^{2}}\left(1-e^{-\xi}\right)-\left(\frac{5}{8 \xi}+\frac{1}{16}-\frac{\xi}{16}\right) e^{-\xi}
$$

where $\operatorname{Ei}(-\xi)$ is a special function known as exponential integral:

$$
E i(-\xi)=-\int_{\xi}^{\infty} \frac{e^{-t}}{t} d t
$$

For a Drude metal, with plasma frequency $\omega_{\mathrm{p}}$ and damping $\gamma$, the electric permittivity $\varepsilon_{\text {bulk }}^{\text {Drude }}$ takes the following expression:

$$
\varepsilon_{b u l k}^{D r u d e}=1-\frac{\omega_{p}^{2}}{\omega(\omega+i \gamma)}
$$

The damping term is inversely proportional to the conductivity $\gamma \propto \frac{1}{\sigma}$, therefore we can derive a thin layer damping $\left(\gamma_{\text {thin }}\right)$ in analogy with the thin layer conductivity:

$$
\frac{\gamma_{\text {thin }}}{\gamma}=\frac{\sigma}{\sigma_{\text {thin }}}=\frac{\Phi(\xi)}{\xi}
$$

and a thin layer Drude dispersion for the electric permittivity $\varepsilon_{\text {thin }}^{\text {Drude }}$

$$
\varepsilon_{\text {thin }}^{\text {Drude }}=1-\frac{\omega_{p}^{2}}{\omega\left(\omega+i \gamma \frac{\Phi(\xi)}{\xi}\right)} .
$$

Unfortunately, close to the plasma frequency most metal, including $\mathrm{Cu}$ and $\mathrm{W}$, do not strictly follow a Drude dispersion. In order to take into account the increasing absorption of thin layers, we subtract from the experimental bulk data taken from the book of Palik ${ }^{19}\left(\varepsilon_{\text {bulk }}^{\text {Palik }}\right)$ the free-electron contribution $\left(\varepsilon_{\text {bulk }}^{\text {Drude }}\right)$ and we subsequently reintroduce the free-electron contribution of the thin layer $\left(\varepsilon_{t h i n}^{\text {Drude }}\right)$ calculated according to Eq.(22):
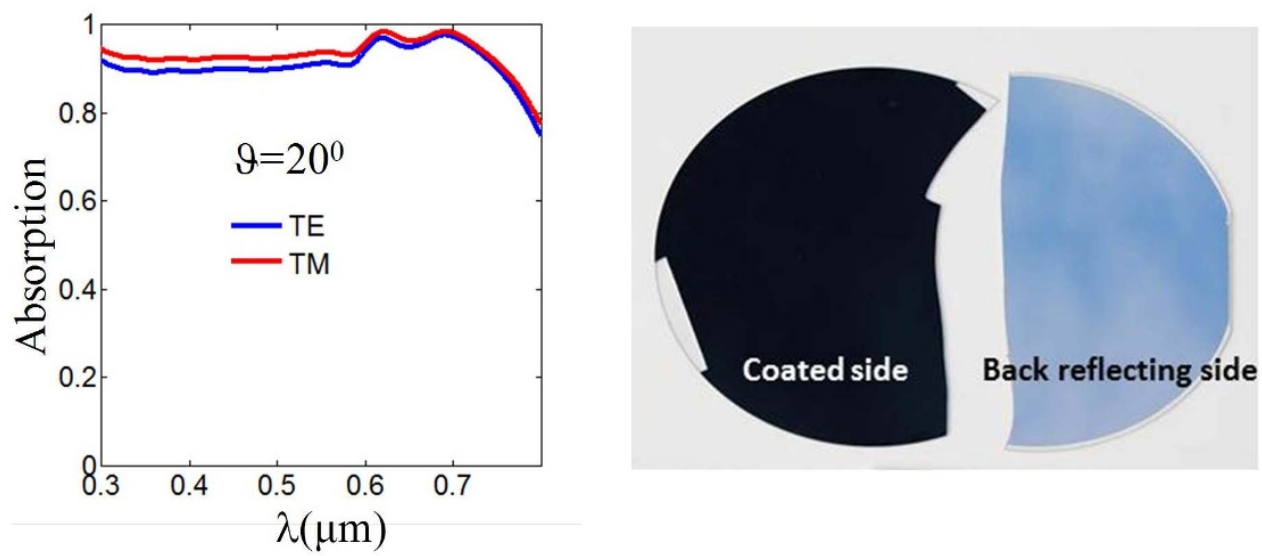

Figure $11 \mid$ [Left] Measured absorption of a $\mathrm{SiO}_{2} / \mathrm{Cu}$ multilayer stack (sample 2) on a reflecting surface made of silver and [Right] picture of the sample taken outdoors under normal daylight. The reflection of the blue sky and clouds is apparent in the picture on the reflecting side of the sample. 

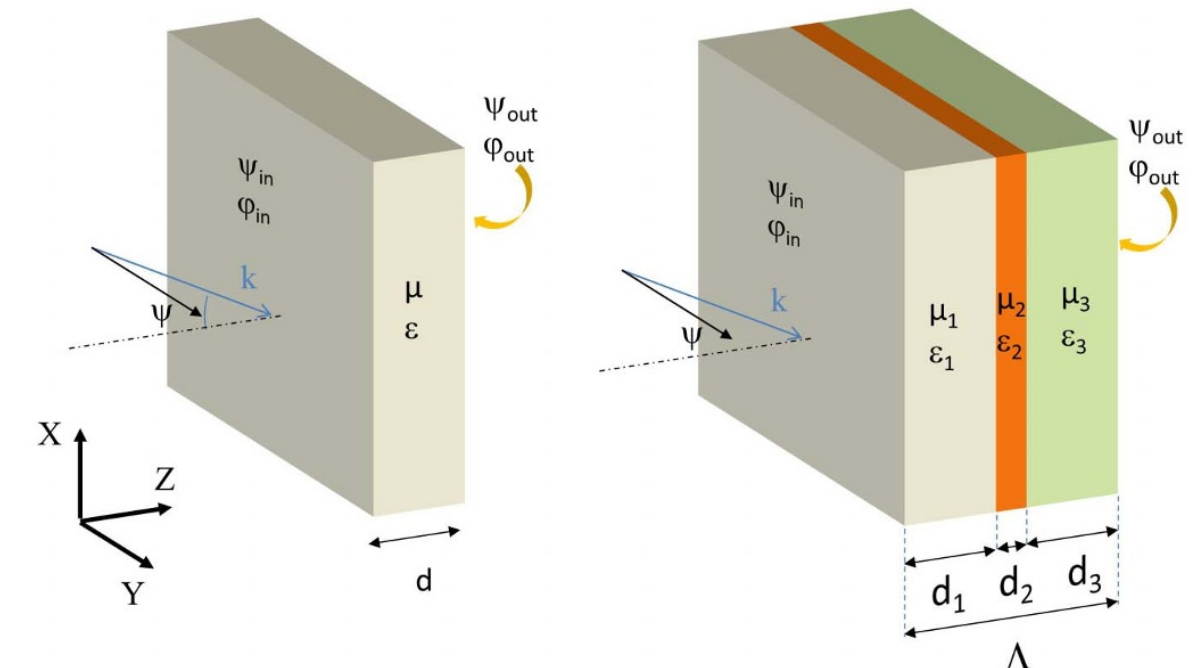

$$
\left(\begin{array}{c}
\Psi_{\text {Out }} \\
\varphi_{\text {Out }}
\end{array}\right)=\left(\begin{array}{cc}
\operatorname{Cos}\left(\mathrm{k}_{\mathrm{z}} \mathrm{d}\right) & \frac{\alpha}{\mathrm{k}_{\mathrm{z}}} \operatorname{Sin}\left(\mathrm{k}_{\mathrm{z}} \mathrm{d}\right) \\
-\frac{\mathrm{k}_{\mathrm{z}}}{\alpha} \operatorname{Sin}\left(\mathrm{k}_{\mathrm{z}} \mathrm{d}\right) & \operatorname{Cos}\left(\mathrm{k}_{\mathrm{z}} \mathrm{d}\right)
\end{array}\right)\left(\begin{array}{c}
\Psi_{I n} \\
\varphi_{I n}
\end{array}\right)
$$

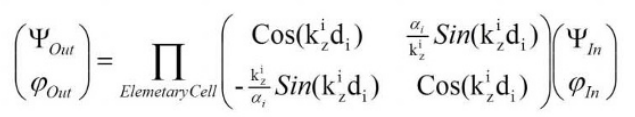

Figure $12 \mid$ Transfer matrix representation for a single layer and a generic three-layer structure.

$$
\varepsilon_{\text {thin }}=\varepsilon_{\text {bulk }}^{\text {Palk }}-\varepsilon_{\text {bulk }}^{\text {Drude }}+\varepsilon_{\text {thin }}^{\text {Drude }} .
$$

Note that in the limit of thick layers, i.e. $\mathrm{d} \gg l$, Eq.(23) gives the correct limit to the experimentally measured data of the bulk dispersion. The electron mean free path for $\mathrm{Cu}$ is $39 \mathrm{~nm}$ and for W $19 \mathrm{~nm}$ at room temperature. according to data reported in literature $e^{26,27}$.

Fabrication. The metal-dielectric stacks were fabricated using a Denton magnetron sputtering system. The $\mathrm{SiO} 2$ film was deposited using a $3^{\prime \prime} \mathrm{SiO}_{2}$ target and the copper layer was deposited using a $3^{\prime \prime}$ copper target. A 700 micron thick quartz wafer was used as a substrate. The system can be used to sputter a 6 " diameter substrate with uniforminity of about $5 \%$ across the substrate.

Optical measurements. Spectral transmission and reflection measurements were done using a Woollam VASE spectroscopic variable angle ellipsometer. The system allows measurements for $\mathrm{s}(\mathrm{TE})$ and $\mathrm{p}(\mathrm{TM})$ polarization from 15 degrees to grazing angle of incidence in reflection and 0 to grazing in transmission. Transmission measurements were taken with respect to no sample in place. Angular measurements were done from 20 to 70 degrees with an increment of 5 degrees.

1. Sergeant, N. P., Agrawal, M. \& Peumans, P. Design of selective coatings for solar thermal applications using subwavelength metal-dielectric structures. Proc. of SPIE 7410, 74100C-1-10 (2009).

2. Min, C. et al. Enhancement of optical absorption in thin-film organic solar cells through the excitation of plasmonic modes in metallic gratings. Appl. Phys. Lett. 96, 133302 (2010).

3. Kaplan, H. Black coatings are critical in optical design. Photonics Spectra 31, 48-50 (1997).

4. Pompea, S. M. \& Breault, R. P. Black surfaces for optical systems, Handbook of Optics, Vol. 2, pp. 37.1-37.63, Ed. by Bass, M., McGraw-Hill, New York, (1995).

5. Stavenga, D. G. Colour in the eyes of insects. J. Comp. Physiol A 188, 337-348 (2002).

6. Vukusic, P., Sambles, J. R. \& Lawrence, C. R. Structurally assisted blackness in butterfly scales. Proc. R. Soc. Lond. B (Suppl.) 271, S237-S239 (2004).

7. Advena, D. J., Bly, V. T. \& Cox, J. T. Deposition and characterization of far infrared absorbing gold black films. Appl. Opt. 32, 1136-1144 (1993).

8. Kodoma, S., Horiuchi, M., Kunii, T. \& Kuroda, K. Ultra-black nickel-phosphorus alloy optical absorber. IEEE Trans. Instrum. Meas. 39, 230-232 (1990).

9. Yang, Z. P., Ci, L., Bur, J. A., Lin, S. Y. \& Ajayan, P. M. Experimental observation of an extremely dark material made by a low-density nanotube array. Nano Letters $\mathbf{8}$, 446-451 (2008).

10. Mizuno, K. et al. A black body absorber from vertically aligned single-walled carbon nanotubes. PNAS 106, 6044-6047 (2009).

11. Shi, H., Ok, J. G., Baac, H. W. \& Guo, L. J. Low density carbon nanotube forest as an index matched and near perfect absorption coating. Appl. Phys. Lett. 99, 211103 (2011).

12. Aydin, K., Ferry, V. E., Briggs, R. M. \& Atwater, H. A. Broadband polarizationindependent resonant light absorption using ultrathin plasmonic super absorbers. Nat. Commun. 2, 517 (2011)
13. Lin, C. H., Chern, R. L. \& Lin, H. Y. Polarization-independent broad-band nearly perfect absorbers in the visible regime. Opt. Expr. 19, 415-424 (2011).

14. Søndergaard, T. et al. Plasmonic black gold by adiabatic nanofocusing and absorption of light in ultra-sharp convex grooves. Nat. Commun. 3, 969 (2012).

15. Narimanov, E. E. \& Kildishev, A. V. Optical black hole: broadband omnidirectional light absorber. Appl. Phys. Lett. 95, 041106 (2009).

16. Avitzour, Y., Urzhumov, Y. A. \& Shvets, G. Wide-angle infrared absorber based on a negative-index plasmonic metamaterial. Phys. Rev. B 79, 045131 (2009).

17. Argyropoulos, C., Le, K. Q., Mattiucci, N., D’Aguanno, G. \& Alu', A. Broadband absorbers and selective emitters based on plasmonic Brewster metasurfaces. Phys. Rev. B 87, 205112 (2013).

18. Mattiucci, N., D’Aguanno, G., Akozbek, N., Scalora, M. \& Bloemer, M. J. Homogenization procedure for a metamaterial and local violation of the second principle of thermodynamics. Opt. Comm. 283, 1613-1620 (2010).

19. Palik, E. D. Handbook of Optical Constants of Solids, Academic Press (1998).

20. Fuchs, K. The conductivity of thin metallic films according to the electron theory of metals. Proc. Cambridge Philos. Soc. 34, 100-108 (1938).

21. Sondheimer, E. H. The mean free path of electrons in metals. Adv. Phys. 1, 1 (1952).

22. Macleod, H. A. Thin film optical filters, (Institute of Physics Publishing, 2001).

23. Lekner, J. Theory of Reflection, Martin Nijhoff Publishers, Dordrecht, 1987.

24. Weiner, A. Ultrafast Optics, John Wiley \& Sons, Hoboken (New Jersey), 2009.

25. Thomson, J. J. On the theory of electric conduction through thin metallic films. Proc. Cambridge Philos. Soc. 11, 120-123 (1901).

26. Sun, T. et al. Dominant role of grain boundary scattering in the resistivity of nanometric Cu films. Phys. Rev. B 79, 041402(R) (2009).

27. Choi, D. et al. Electron mean free path of tungsten and the electrical resistivity of epitaxial (110) tungsten films. Phys. Rev. B 86, 045432 (2012).

\section{Acknowledgments}

This work has been supported by the Defense Advanced Research Agency (DARPA) with SBIR W31P4Q-11-C-0109, by the Army Research Office (ARO) with STTR W31P4Q-11-C-0098, and by AEgis Technologies with an IRAD (Internal Research and Development) grant to G.D. We thank Shelby Hempstead for help in the preparation of the samples.

\section{Author contributions}

N.M. and G.D. conceived the idea, developed the analytical model, contributed to the writing of the paper and the interpretation of the experimental results, M.J.B. contributed to the interpretation of the experimental results and the writing of the paper, N.A. contributed to the writing of the paper, measurements and the preparation of the samples.

\section{Additional information}

Competing financial interests: The authors declare no competing financial interests. 
How to cite this article: Mattiucci, N., Bloemer, M.J., Aközbek, N. \& D’Aguanno, G. Impedance matched thin metamaterials make metals absorbing. Sci. Rep. 3, 3203; DOI:10.1038/srep03203 (2013). (c) (i) $(-)$ This work is licensed under a Creative Commons AttributionBy No No NonCommercial-NoDerivs 3.0 Unported license. To view a copy of this license, visit http://creativecommons.org/licenses/by-nc-nd/3.0 